\title{
Numerical modelling of bubble coalescence and droplet separation
}

\author{
Y. Y. Yan \& Y. Q. Zu \\ School of the Built Environment, University of Nottingham, UK
}

\begin{abstract}
Both bubble coalescence and droplet separation are important physical phenomena in the natural world and a variety of process industries. This paper presents results of numerical simulation of behaviours of bubble coalescence and droplet separation. The velocity distribution functions of two particles are used in lattice Boltzmann equations. Based on the lattice Boltzmann method (LBM), both the phenomena of two rising bubbles coalescing in liquid and a liquid droplet break-up on wetting boundaries are simulated. Typically, such two-phase problems of large ratio of liquid-gas densities up to 1000 are studied.

Keywords: bubble coalescence, droplet separation, numerical modelling, lattice Boltzmann method.
\end{abstract}

\section{Introduction}

Both bubble coalescence and droplet separation are important physical phenomena in the natural world and a variety of process industries. It is a common occurrence in two phase flow and flow boiling that the evolution of bubbly flow to slug and annular flows accompanies processes of bubble coalescences. The coalescence or separation of droplets are also popular in droplet and film cooling condensations under difference surface conditions. Numerical modelling of bubble coalescence or droplet separation has been attempted by researchers for many years. Conventional CFD methods based on solving Navier-stokes equations can simulate free surface flow and bubble shape evolution with time [1-3] but can not effectively simulate problems of bubbles or droplets coalescences. Although the phenomena of bubble coalescences have been simulated by VOF method in [4] but only a two dimensional problem was discussed and the ratio of gas-liquid densities was also limited. 
In recent years, the lattice Boltzmann method (LBM) has become an established numerical scheme for simulating multiphase fluid flows. Several researchers have applied LBM to study multiphase flow including bubbles or droplets coalescence [5-7]. The key idea behind the LBM is to recover the correct macroscopic motion of fluid by incorporating the complicated physics of the problem into simplified microscopic models or mesoscopic kinetic equations. In this method, kinetic equations for particle velocity distribution function are solved; and macroscopic quantities are then obtained by evaluating hydrodynamic moments of the distribution function. LBM has many computational advantages, such as parallel of algorithm and simplicity of programming [8]. In LBM modelling of multiphase fluid flows, Gunstensen et al. [9] developed a multi-component model based on the two-component lattice gas model; Shan and Chen [10] presented a model with mean-field interactions for multi-phase/component fluid flows; Swift et al. [11, 12] proposed a LBM model for multi-phase flows using the idea of free energy; He et al. [13] also developed a model using the index function to track the interface of multi-phase flow. To overcome the shortcoming that the above LBM schemes can only simulate two-phase fluids with small density ratios (less than 20) due to numerical instability, Inamuro et al. [6] proposed a LBM for incompressible twophase flows with large density differences by using the projection method. Briant et al. [14-15] developed an approach based on the free energy model introduced in $[11,12]$ to simulate partial wetting and contact line motion in twophase fluids. However, as the method naturally inherits the disadvantage of original free energy model of Swift et al. [11, 12] and can only be used to simulate problems with a small density ratio which was around 2 [14].

In the present paper, based on a new LBM scheme developed in [16], both bubble coalescence in liquid with unconfined boundary and a liquid droplet separation on a wetting boundary are studied.

\section{The Lattice Boltzmann Model}

Based on the three-dimensional nine-velocity (D3Q15) LBM model, as shown in Fig. 1, the particle velocity in the $\alpha$ th direction, $\vec{e}_{\alpha}$, is given by [6]

$$
\begin{aligned}
& {\left[\mathbf{e}_{0}, \mathbf{e}_{1}, \mathbf{e}_{2}, \mathbf{e}_{3}, \mathbf{e}_{4}, \mathbf{e}_{5}, \mathbf{e}_{6}, \mathbf{e}_{7}, \mathbf{e}_{8}, \mathbf{e}_{9}, \mathbf{e}_{10}, \mathbf{e}_{11}, \mathbf{e}_{12}, \mathbf{e}_{13}, \mathbf{e}_{14}\right]} \\
& =\left[\begin{array}{ccccccccccccccc}
0 & 1 & 0 & 0 & -1 & 0 & 0 & 1 & -1 & 1 & 1 & -1 & 1 & -1 & -1 \\
0 & 0 & 1 & 0 & 0 & -1 & 0 & 1 & 1 & -1 & 1 & -1 & -1 & 1 & -1 \\
0 & 0 & 0 & 1 & 0 & 0 & -1 & 1 & 1 & 1 & -1 & -1 & -1 & -1 & 1
\end{array}\right]
\end{aligned}
$$

To simulate a two-phase flow problem, two velocity distribution functions of particles, $f_{\alpha}$ and $g_{\alpha}$, are introduced. Function $f_{\alpha}$ is used to calculate the order parameter, $\phi$, which distinguishes two phases, and function $g_{\alpha}$ is used to calculate the predicted velocity, $\vec{u} *$. 
The evolution of particle distribution functions $f_{\alpha}(x, t)$ and $g_{\alpha}(x, t)$ with particle velocity $\vec{e}_{\alpha}$ at point $x$ and time $t$ is calculated by following equations:

$$
\begin{aligned}
& f_{\alpha}\left(\vec{x}+\vec{e}_{\alpha} \delta_{t}, t+\delta_{t}\right)=f_{\alpha}^{(e q)}(\vec{x}, t) \\
& g_{\alpha}\left(\vec{x}+\vec{e}_{\alpha} \delta_{t}, t+\delta_{t}\right)=g_{\alpha}^{(e q)}(\vec{x}, t)
\end{aligned}
$$

where $\vec{u}, \rho$ and $\mu$ are the macroscopic velocity, density and dynamic viscosity respectively; $\delta_{t}=1$ is the time step during which the particles travel the lattice spacing; $f_{\alpha}^{(e q)}$ and $g_{\alpha}^{(e q)}$ are the corresponding equilibrium states of $f_{\alpha}$ and $g_{\alpha}$, which have been given in details in [16].
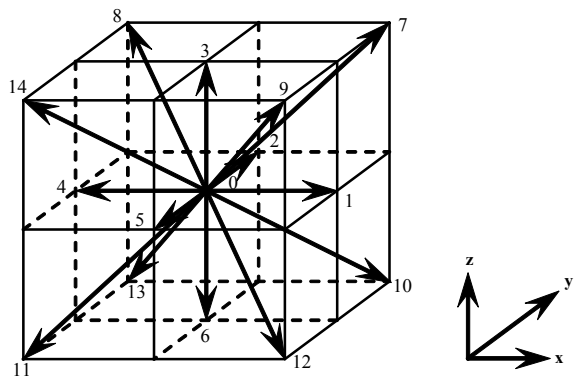

Figure 1: Discrete velocity set of three-dimensional fifteen-velocity model.

The macroscopic quantities, $\vec{u}^{*}, \phi, \rho, \mu$ in the LBM can be evaluated as

$$
\begin{aligned}
& \phi=\sum_{\alpha} f_{\alpha}, \quad \vec{u}^{*}=\sum_{\alpha} \vec{e}_{\alpha} g_{\alpha}
\end{aligned}
$$

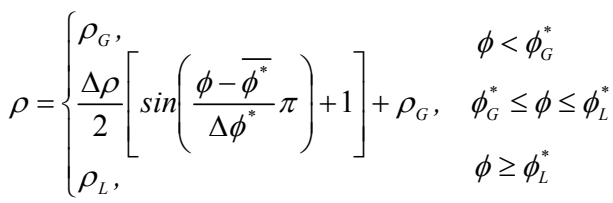

$$
\begin{aligned}
& \mu=\frac{\rho-\rho_{G}}{\rho_{L}-\rho_{G}}\left(\mu_{L}-\mu_{G}\right)+\mu_{G}
\end{aligned}
$$

where $\phi_{L}^{*}$ and $\phi_{G}^{*}$ are the cut-off values of the order parameter, $\rho_{L}$ and $\rho_{G}$ are the density of liquid and gas phases respectively. $\mu_{L}$ and $\mu_{G}$ are the dynamic viscosity of liquid and gas phases respectively. $\Delta \rho=\rho_{L}-\rho_{G}, \Delta \phi^{*}=\phi_{L}^{*}-\phi_{G}^{*}$ and $\overline{\phi^{*}}=\left(\phi_{L}^{*}+\phi_{G}^{*}\right) / 2$.

To obtain the velocity field which satisfies the continuity equation $(\nabla \cdot \mathrm{u}=0)$, the predicted velocity $\vec{u} *$ is corrected by using the following equations, 


$$
\begin{gathered}
\frac{\vec{u}-\vec{u}^{*}}{\delta_{t}}=-\frac{\nabla p}{\rho} \\
\nabla \cdot\left(\frac{\nabla p}{\rho}\right)=\frac{\nabla \cdot \vec{u}^{*}}{\delta_{t}}
\end{gathered}
$$

where $p$ is the pressure of the two-phase/component fluid, which can be obtained by solving Eq. (8) in the following LBM framework for velocity distribution function:

$$
h_{\alpha}\left(\vec{x}+\vec{e}_{\alpha}, n+1\right)=h_{\alpha}(\vec{x}, n)-\frac{1}{\tau}\left[h_{\alpha}(\vec{x}, n)-\omega_{\alpha} p(\vec{x}, n)\right]-\frac{\omega_{\alpha}}{3} \frac{1}{\rho} \nabla \cdot \vec{u} *
$$

where $n$ is the number of iterations, $\omega_{\alpha}$ is wetting coefficient and $\tau=0.5+1 / \rho$ is a relaxation time. The pressure at step $n+1$ is given by

$$
p(\vec{x}, n+1)=\sum_{\alpha} h_{\alpha}(\vec{x}, n+1)
$$

The convergent pressure $p$ is determined when

$$
\forall \vec{x} \in V,|p(\vec{x}, n+1)-p(\vec{x}, n+1)|<\varepsilon
$$

where $V$ denotes the whole computational domain. Substituting the newly obtained pressure $p$ into and solving Eq. (7) gives the corrected velocity field $\vec{u}$.

As stated and demonstrated in $[6,16]$, this method can be used to simulate two-phase flow with density ratio up to 1000 .

\section{Wetting boundary condition}

The liquid-gas surface tension force $\sigma_{L G}$ is given in [17] as

$$
\sigma_{L G}=\frac{\left(\phi_{L}-\phi_{G}\right)^{3}}{6} \sqrt{2 k_{f} \beta}
$$

where $\phi_{L}$ and $\phi_{G}$ are the order parameters of liquid and gas, respectively; $k_{f}$ is a constant parameter to decide the width of interface and the strength of surface tension; $\beta$ is the constant relating to interfacial thickness. According to Young's law [18], when a liquid-gas interface meets a partial wetting solid wall, the contact angle, $\theta_{w}$, measured in the liquid, can be calculated from a balance of surface tension forces at the contact line as 


$$
\cos \theta_{w}=\frac{\sigma_{S G}-\sigma_{S L}}{\sigma_{L G}} ;
$$

where $\sigma_{S G}$ and $\sigma_{S L}$ are the solid-gas and solid-liquid surface tensions, respectively, which can be represented as [16]: If $\lambda>0$,

$$
\begin{gathered}
\sigma_{S G}=-\lambda \phi_{2}+\int_{\phi_{G}}^{\phi_{2}} \sqrt{2 k_{f} \psi} d \phi=-\lambda \frac{\phi_{L}+\phi_{G}}{2}+\frac{\sigma_{L G}}{2}-\frac{\sigma_{L G}}{2}(1-\Omega)^{3 / 2}, \\
\sigma_{S L}=-\lambda \phi_{4}+\int_{\phi_{L}}^{\phi_{4}} \sqrt{2 k_{f} \psi} d \phi=-\lambda \frac{\phi_{L}+\phi_{G}}{2}+\frac{\sigma_{L G}}{2}-\frac{\sigma_{L G}}{2}(1+\Omega)^{3 / 2} ;
\end{gathered}
$$

and if $\lambda<0$,

$$
\begin{aligned}
& \sigma_{S G}=-\lambda \phi_{1}+\int_{\phi_{1}}^{\phi_{G}} \sqrt{2 k_{f} \psi} d \phi=-\lambda \frac{\phi_{L}+\phi_{G}}{2}+\frac{\sigma_{L G}}{2}-\frac{\sigma_{L G}}{2}(1-\Omega)^{3 / 2}, \\
& \sigma_{S L}=-\lambda \phi_{3}+\int_{\phi_{3}}^{\phi_{L}} \sqrt{2 k_{f} \psi} d \phi=-\lambda \frac{\phi_{L}+\phi_{G}}{2}+\frac{\sigma_{L G}}{2}-\frac{\sigma_{L G}}{2}(1+\Omega)^{3 / 2} ;
\end{aligned}
$$

where $\phi_{i=1,2,3,4}$ are the solutions of order parameter; $\psi$ is the free energy density, and $\Omega$ the wetting potential given by

$$
\Omega=\frac{4 \lambda}{\left(\phi_{L}-\phi_{G}\right)^{2} \sqrt{2 k_{f} \beta}}
$$

where $\lambda= \pm \sqrt{2 k_{f} \psi\left(\phi_{s}\right)}, \phi_{s}$ is the order parameter at solid wall [16]. The wetting angle can be determined by substituting Eq. (12) and Eqs. (14)-(17) into Eq. (13) and written as

$$
\cos \theta_{w}=\frac{(1+\Omega)^{3 / 2}-(1-\Omega)^{3 / 2}}{2}
$$

For a given wetting angle at $0<\theta_{w}<\pi, \Omega$ can be obtained from Eq. (19) as,

$$
\Omega=2 \operatorname{sgn}\left(\frac{\pi}{2}-\theta_{w}\right)\left\{\cos \left(\frac{\gamma}{3}\right)\left[1-\cos \left(\frac{\gamma}{3}\right)\right]\right\}^{1 / 2}
$$

where $\gamma=\arccos \left(\sin ^{2} \theta_{w}\right)$ and $\operatorname{sgn}(\xi)$ gives the sign of $\xi$. It is noted from Eq. (20) that the required wetting potential $\Omega$ can be obtained by choosing a desired contact angle $\theta_{w}$ and then calculating $\lambda$ by Eq. (18) with a newly obtained $\Omega$.

In order to introduce the partial wetting boundary condition to the LBM simulation through imposing it through equilibrium distribution functions $f_{\alpha}^{(e q)}$ and $g_{\alpha}^{(e q)}$, the following boundary conditions should be imposed: 


$$
\begin{gathered}
\left.\frac{\partial \phi}{\partial z}\right|_{z=0}=-\frac{\lambda}{k_{f}}, \\
\left.\frac{\partial^{2} \phi}{\partial z^{2}}\right|_{z=0} \approx \frac{1}{2}\left(-\left.3 \frac{\partial \phi}{\partial z}\right|_{z=0}+\left.4 \frac{\partial \phi}{\partial z}\right|_{z=1}-\left.\frac{\partial \phi}{\partial z}\right|_{z=2}\right)
\end{gathered}
$$

where $z$ is the perpendicular direction to the wall. In this scheme, Eq. (21) is used to determine the first term on the right hand side of Eq. (22). While the second term is calculated using a standard centred finite-difference formula. Finally, Briant et al. [14] found empirically that the best choice for the third term is a left-handed finite-difference formula taken back into the wall, i.e.

$$
\left.\frac{\partial \phi}{\partial z}\right|_{z=2} \approx \frac{1}{2}\left(\left.3 \phi\right|_{z=2}-\left.4 \phi\right|_{z=1}+\left.\phi\right|_{z=0}\right)
$$

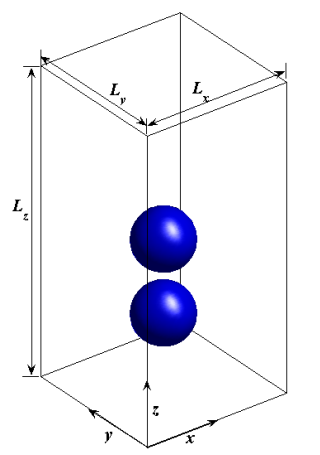

Figure 2: Computational domain.

\section{Results and discussion}

\subsection{Bubble coalescence in unbounded liquid}

The method is firstly applied to bubble coalescence; the coalescence of two rising bubbles is simulated and two cases are calculated. The computational domain is shown in Fig. 2. In an initial study, two bubbles with the same diameter $D$ are placed $5 D / 4$ apart in a liquid inside a rectangular domain and is released at time $t=0$. Calculations are carried out for the cases of liquid and gas phases with different density ratios, $\rho_{L} / \rho_{G}$, and viscosity ratios, $\mu_{L} / \mu_{G}$. Dimensionless parameters, Morton number: $M=g \mu_{L}^{4}\left(\rho_{L}-\rho_{G}\right) /\left(\rho_{L}^{2} \sigma^{3}\right)$ and Eötvös number: $E=g\left(\rho_{L}-\rho_{G}\right) D^{2} / \sigma^{3}$ are applied for the simulated phenomena. Periodic boundary conditions are imposed on all sides of the computational domain, which is divided into $64 \times 64 \times 128$ cubic lattice. The diameter of each initial bubble occupies 24 lattice spaces, i.e. $D=24 \delta x$. The 
behaviour of the two bubbles evolutes with time, typically how the lower bubble catches up and finally coalesces with the upper bubble is studied. Velocity vectors of both inside and around the bubbles during the evolution are also studied.

Fig. 3 shows time evolution of two bubble coalescence and velocity vectors at section of $y=L_{y} / 2$. The two gas bubbles rising in an unbounded liquid with $\rho_{L} / \rho_{G}=50, \mu_{L} / \mu_{G}=50$, dimensionless Morton number $M=1 \times 10^{-5}$, and Eötvös number $E=10$ are simulated; where $t^{*}$ refers to dimensionless time $\left(t^{*}=t U / D\right)$, here $U$ is the terminal averaged velocity of gas phase.

Fig. 4 shows two bubble coalescence when gas bubbles rise in an unbounded liquid at $\rho_{L} / \rho_{G}=1000, \mu_{L} / \mu_{G}=50$, Morton number $M=1$, and Eötvös number $E=15$. The upper figure shows time evolution of bubble shapes and the lower figure shows the velocity vectors at section of $y=L_{y} / 2$;

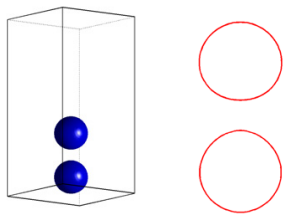

$t^{*}=0$

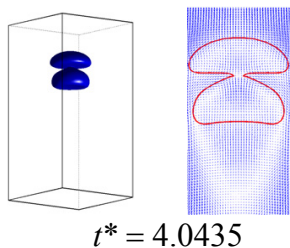

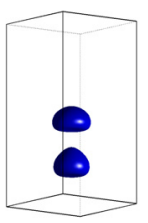

$t^{*}=1.3647$

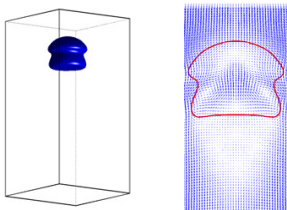

$t^{*}=4.4816$
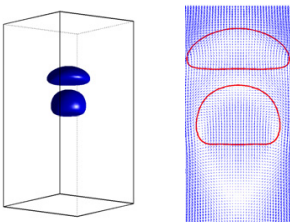

$t^{*}=3.3696$

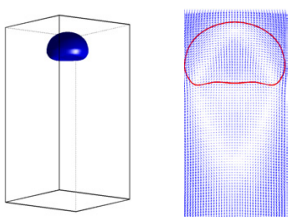

$t^{*}=4.8859$

Figure 3: Coalescence of two rising bubbles in liquid with $\rho_{L} / \rho_{G}=50$, $\mu_{L} / \mu_{G}=50, M=1 \times 10^{-5}, E=10$.

\subsection{Droplets separation on a wetting boundary}

The method is applied to calculate a water droplet spreading on a uniform wetting surface. Initially, as shown in Fig. 5, the shape of droplet is spherical, the distance between the centre of the sphere and the wall is $r=1 \times 10^{-3} \mathrm{~m}$, where $r$ is the radius of the initial droplet. The computational domain is filled with air except the location occupied by the water and is divided into $120 \times 100 \times 40$ uniform cubic lattices.

The motion of water droplets at normal temperature surrounded by air on partial wetting walls is considered. Naturally, the densities of two fluids are set at $\tilde{\rho}_{L}=1 \times 10^{3} \mathrm{~kg} / \mathrm{m}^{3}, \tilde{\rho}_{G}=1.29 \mathrm{~kg} / \mathrm{m}^{3}$, and meanwhile the dynamic viscosities of them are at $\tilde{\mu}_{L}=1 \times 10^{-3} \mathrm{~kg} / \mathrm{ms}, \tilde{\mu}_{G}=1.935 \times 10^{-5} \mathrm{~kg} / \mathrm{ms}$, respectively. The initial surface tension between water and air is $\widetilde{\sigma}_{L G}=1 \times 10^{-3} \mathrm{~kg} / \mathrm{s}^{2}$ and the gravitational 
acceleration is set at $\widetilde{g}=9.8 \mathrm{~m} / \mathrm{s}^{2}$. To relate the physical parameters with simulation parameters, a length scale of $L_{0}=1 \times 10^{-4} \mathrm{~m}$, a time scale of $T_{0}=1 \times 10^{-6} \mathrm{~s}$ and a mass scale of $M_{0}=1 \times 10^{-12} \mathrm{~kg}$ are chosen; these lead to the dimensionless parameters: $\rho_{L}=1 \times 10^{3} ; \rho_{G}=1.29 ; \mu_{L}=0.1 ; \mu_{G}=1.935 \times 10^{-3}$; $\phi_{L}=0.4 ; \quad \phi_{G}=0.1 ; k=0.05 ; g=9.8 \times 10^{-8}$. Unless otherwise specified, the flowing simulations are within a cuboid computational domain with a no-slip boundary at the lower surface, i.e. the flat partial wetting wall, and the free outflow/inflow boundaries at the other five surfaces. $\varepsilon$ in Eq. (11) is set as $\varepsilon=1 \times 10^{-6}$.
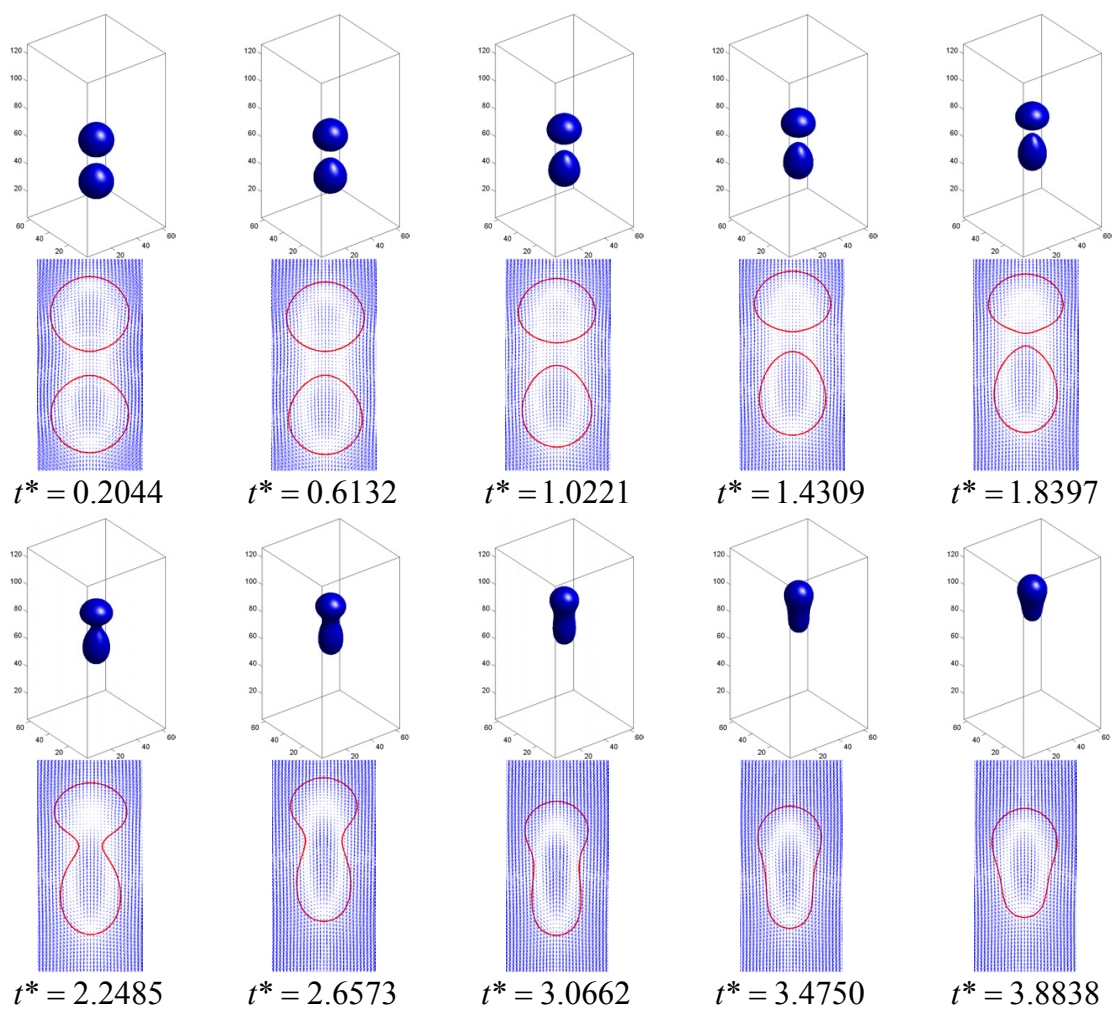

Figure 4: Time evolution of bubble shapes and Velocity vectors at section of $y=L_{y} / 2$ of coalescence of two rising bubbles in liquid with $\rho_{L} / \rho_{G}=1000, \mu_{L} / \mu_{G}=50, M=1, E=15$.

Fig. 6 shows how a small hemispherical water droplet evolves with time on a heterogeneous surface. A narrow hydrophobic strip with width of $l=6 \times 10^{-4} \mathrm{~m}$ is located at the centreline of the surface where $\theta_{w}=5 \pi / 6$, and the other area is 
occupied by the hydrophilic surface with $\theta_{w}=\pi / 6$. The initial droplet which has a radius $r=1.5 \times 10^{-3} \mathrm{~m}$ is set at the centre of the wetting surface. As shown in Fig. 6, the droplet stretches over the area occupied by the hydrophilic surface in the early stages of flow evolution due to the adhesive force of the surface. At the same time, the droplet rapidly contracts inward along the hydrophobic strip. With the development of time, the droplet spreads further on the hydrophilic area, and meanwhile contracts inward along the hydrophobic strip and finally breaks up into two smaller droplets. The newly formed droplets continue spreading until an equilibrium state is reached. For a uniform hydrophilic surface separated by a hydrophobic strip, the spreading dynamics of the droplet is affected by three parameters, namely, the width of the hydrophobic strip, the gravity and the wetting property of the hydrophilic surface [19]. A further examination and analysis of the effects of these three parameters on the spreading and break-up of the droplet will be done in the near future.

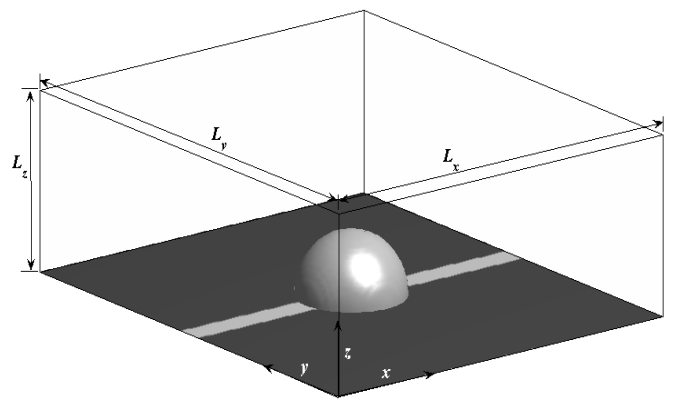

Figure 5: Computational domain.

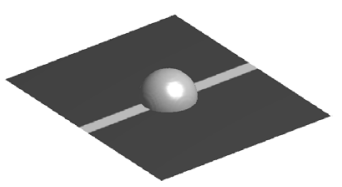

$t=0.0 \mathrm{~s}$

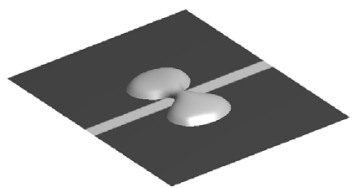

$t=0.15 s$

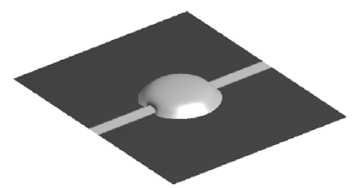

$t=0.015 s$

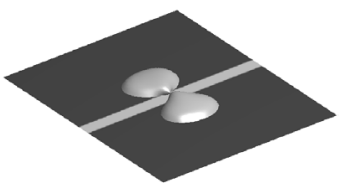

$t=0.152 s$

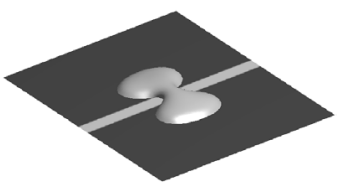

$t=0.14 s$

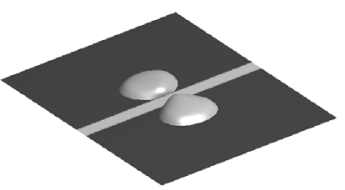

$t=0.154 s$

Figure 6: Snapshots of droplet spreading and its break-up on heterogeneous surface. 


\section{Conclusions}

A lattice Boltzmann method which can simulate two-phase fluids with large density ratio, and meanwhile deal with interactions between a fluid-fluid interface and a partial wetting wall is developed. Based on this method, the dynamics of two rising bubbles in a liquid with liquid-gas density up to 1000 is simulated. In addition, it is also simulated that a liquid drop breaks up on uniform and heterogeneous walls with liquid-gas density ratio of 1000:1.29. The results of simulations can generally confirm that the current LBM is suitable to study such two-phase flow problems with high ratios of liquid-gas densities and with such partially wetting boundaries. A further experimental validation of the numerical method will be carried out in the near future.

\section{Acknowledgement}

The project is supported by British EPSRC under grant EP/D500125/1.

\section{References}

[1] Ryskin, G. and Leal, L.G., 1984, Numerical solution of free-boundary problems in fluid mechanism, Part 1, Finite-Difference Technique, J. Fluid Mech., 148, 1-17.

[2] Li, W.Z., Yan, Y.Y. and Smith, J.M., 2003, A numerical study of the interfacial transport characteristics outside spheroidal bubbles and solids. Int. J. of Multiphase Flow, 29(3), 435-460.

[3] Yan, Y.Y. and Li, W.Z., 2006. Numerical modelling of a vapour bubble growth in uniformly superheated liquid, Int. J. of Numerical Methods for Heat \& Fluid Flow, 16(7), 764-778.

[4] Krishna, R. and Baten, J.M. van, 1999, Simulating the motion of gas bubbles in a liquid, Nature, Vol. 398, 208.

[5] Yang, Z.L., Do, Q.M., Palm, B. and Sehgal, B.R., 2000, Numerical simulation of bubble dynamics: lattice Boltzmann approach, Proc. of $5^{\text {th }}$ Int. Symp. on Heat Transfer, 12-16 Aug., Beijing, 598-603.

[6] Inamuro, T., Ogata, T., Tajima, S. and Konishi, N., 2004, A lattice Boltzman method for incompressible two-phase flows with large density differences. J. Comput. Phys., 198, 628-644.

[7] Zheng, H.W., Shu, C. and Chew, Y.T., 2006, A lattice Boltzmann model for multiphase flows with large density ratio, J. of Compt. Phys., 218, 353-371.

[8] Chen, S. and Doolen, G.D., 1998, Lattice Boltzmann method for fluid flows. Annual Review of Fluid Mechanics 30, 329-364.

[9] Gunstensen, A.K., Rothman, D.H., Zaleski, S. and Zanetti, G., 1991, Lattice Boltzmann Model of Immiscible Fluids. Phys. Rev. A 43, 43204327. 
[10] Shan, X.W. and Chen, H.D., 1993, Lattice Boltzmann Model for Simulating Flows with Multiple Phases and Components. Phys. Rev. E 47,1815-1819.

[11] Swift, M.R., Osborn, W.R. and Yeomans, J.M., 1995, Lattice Boltzmann Simulation of Nonideal Fluids. Phys. Rev. Lett. 75, 830-833.

[12] Swift, M.R., Orlandini, E., Osborn, W.R. and Yeomans, J.M., 1996, Lattice Boltzmann simulations of liquid-gas and binary fluid systems, Phys. Rev. E 54, 5041-5052.

[13] He, X.Y., Chen, S.Y. and Zhang, R.Y., 1999, A lattice Boltzmann scheme for incompressible multiphase flow and its application in simulation of Rayleigh-Taylor instability, J. of Compt. Phys. 152, 642-663.

[14] Briant, A.J., Papatzacos, P. and Yeomans, J.M., 2002, Lattice Boltzmann simulations of contact line motion in a liquid-gas system. Phil. Trans. of the Royal Society Series a-Mathematical Phys. and Eng. Sci. 360, 485495.

[15] Dupuis, A. and Yeomans, J.M., 2005, Modeling droplets on superhydrophobic surfaces: Equilibrium states and transitions. Langmuir 21, 2624-2629.

[16] Yan, Y.Y. and Zu, Y.Q., 2006, A lattice Boltzmann method for incompressible two-phase flows on partial wetting surface with large density ratio, submitted to J. Compt. Phys.

[17] Rowlinson, J.S. and Widom, B., 1989, Molecular Theory of Capillarity, Clarendon, Oxford.

[18] Young, T., 1805, An essay on the cohesion of fluids. Phi. Trans. R. Soc. Lond. 95, 65-87. 